Original Paper $\quad$ http://ajol.info/index.php/ijbcs $\quad$ http://indexmedicus.afro.who.int

\title{
Composition and dynamic of benthic macroinvertebrates community in semi-arid area rivers of Burkina Faso (West Africa)
}

\author{
Idrissa KABORÉ ${ }^{1,2^{*}}$, Idrissa OUÉDRAOGO ${ }^{2}$, Lallébila TAMPO ${ }^{3}$, Adama OUÉDA $^{2}$, \\ Otto MOOG ${ }^{1}$, Wendengoudi GUENDA ${ }^{2}$ and Andreas H. MELCHER ${ }^{1}$
}

\author{
${ }^{1}$ Department of Water, Atmosphere and Environment, Institute of Hydrobiology and Aquatic Ecosystem \\ Management, BOKU University of Natural Resources and Life Sciences, Max Emmanuel Strasse 17, 1180 \\ Vienna, Austria. \\ ${ }^{2}$ Université de Ouagadougou, UFR-Sciences de la Vie et de la Terre, Laboratoire de Biologie et Ecologie \\ Animales (LBEA), Burkina Faso, 03 BP 7021 Ouagadougou 03, Burkina Faso. \\ ${ }^{3}$ Laboratory of Waters Chemistry, Sciences Faculty, University of Lomé, BP1515, Togo. \\ *Corresponding author; E-mail: ikabore16@yahoo.fr
}

\begin{abstract}
The benthic macroinvertebrates communities dynamic were investigated in rivers from Burkina Faso in the purpose to analyze the taxonomic composition, the structure of benthic macroinvertebrates community and the composite environmental variables that correspond to the major distribution patterns of this community. The results showed that a total of 132 taxa was recorded and the large majority of these (103 taxa) belonged to 57 families from 8 orders of insects that represent $95 \%$ of relative abundance. We also observed some distinct differences relative to the spatial and temporal variation in the taxonomic composition. The canonical correspondance analysis (CCA) revealed a strong correlationship between Chironomidae, Syrphidae, Culicidae, Psychodidae, as well as the Pulmonates molluscs and organic nutriments feeding dynamics. These findings showed the sensitivity of benthic macroinvertebrates at different level: sensitivity which could be attributable to man-induced activities.
\end{abstract}

(C) 2016 International Formulae Group. All rights reserved.

Keywords: Benthic macroinvertebrates, enviromental variables, dynamic, Burkina Faso.

\section{INTRODUCTION}

The benthic macroinvertebrates assemblages are widely recognised as the most important group living in rivers and lakes (Covich et al., 1999). They play a key role for the understanding of the structure and functioning of these rivers and lakes ecosystems, due to their wide distribution and limited migration ability and resiliency
(Barbour et al., 1999). The benthic macroinvertebrates have been widely used in biomonitoring program, due to several attributes that make them particularly beneficial (Moog et al., 1999; Marzin, 2013). In Addition, they are a primary food source for many fish species, amphibians and birds (Voshell and Reese, 2002). Despite the benefits and the services that they provide to 
humans, waters quality and its living organisms are threatened by human activities such as rapid expansion of urban areas and agriculture (Moore and Palmer, 2005; Kaboré et al., 2016). These pressures caused many changes in the structure of macroinvertebrates community and lead to the decline of biodiversity due to habitat fragmentations and the water pollution in many developing countries. Though, in tropical area, specially in West Africa, the knowledge on these organisms and their ecology is still fragmentary (Sharma et al. 1993; George et al., 2010; Camara et al., 2012; Mesa et al., 20013; Edia et al., 2013). In Burkina Faso, only a few studies have been conducted with benthic macroinvertebrate communities. These studies mainly concerned the inventory of macroinvertebrates in the Mouhoun river (Guenda, 1985, 1996) and the response of benthic macroinvertebrates to anthropogenic interferences (Koblinger and Trauner, 2013; Sanogo et al., 2014; Kaboré et al., 2016). However, the spatial and temporal dynamic changes in macroinvertebrates were not clearly addressed in these earlier studies. The key aims of the present study is to identify and describe the composition, the diversity of benthic macroinvertebrates in Burkina Faso and to determine the enviromental factors that influence the macroinvertebrates distribution.

\section{MATERIALS AND METHODS Study area}

The study was undertaken in three main rivers in Burkina Faso described in Kaboré et al. (2016): Volta rivers (Mouhoun and Nakambé) and Comoé river (Figure 1). Volta river covers an estimated area of 400,000 $\mathrm{km}^{2}$ and spread over six West African countries (Sanwidi, 2007). Located between the north latitudes of $5^{\circ} 30^{\prime} \mathrm{N}$ in Ghana $14^{\circ} 30^{\prime} \mathrm{N}$ in Mali and the longitudes $5^{\circ} 30^{\prime} \mathrm{W}$ to $2^{\circ} 00^{\prime} \mathrm{E}$, the Volta rivers basin is drained mainly by two main sub-basins in the northern part which belong to Burkina Faso. In Burkina Faso the Volta rivers names changed to Nakanbé (formerly White Volta) and the Mouhoun (formerly Black Volta). Natural vegetation, mostly savanna grasslands, uses the major part of the rainfall (around $80 \%$ ) throughout the basin. River discharge is highly sensitive to variations in annual rainfall (Sanwidi, 2007). The geological formations of the basin are dominated by the Voltaian system consisted of Precambrian to Paleozoic sandstones, shales and conglomerates. Water resources and agricultural activities in the basin are therefore unpredictable. However, 18.6 million people (Barry et al., 2005; Sanwidi, 2007 ) with an annual growth rate of $2.4 \%$ rely on these activities.

The Comoé (area of 17,590 $\mathrm{km}^{2}$ covering $6.4 \%$ ) is a perennial river in the extreme south-west of Burkina Faso where the annual rainfall exceeds $1000 \mathrm{~mm}$ (Sally et al., 2011) with annual evaporation rate of 2000 mm (Kabré et al., 2002). Bordering Mali and Ivory Coast, it is located in the Sudanian climatic zone with tropical characteristics (Sirima et al., 2009). Temperatures in the basin vary, from 24 to $25^{\circ} \mathrm{C}$ minimal and 31 to $32{ }^{\circ} \mathrm{C}$ the maximal. The area drained by Comoé river is called "Cascades" due to the abundance of water. Dams were also constructed in the area to secure sugar production and also guarantee the municipal water supply to the town of Banfora (Sally et al., 2011). The basin is drained by the Léraba and Comoé rivers, which are perennial, and by several temporary rivers such as Kodoum, Baoué and Iringou. The total annual surface discharge is estimated at 1.6 billion cubic meters of which 85 million cubic meters are retained by dams.

Sampling sites lay within a continuum ranging from low to very high intensity of anthropogenic impacts in the floodplain area (Kaboré et al., 2016). Three sampling campaigns were conducted according to the rivers hydrology. The first sampling was conducted in 2012 from July to September corresponding to high water flow (rainy reason), and low water flow (end of rainy season) from October to December of the same year. The third sampling was conducted 
from March to June 2014, corresponding at lowest water flow (dry season).

\section{Physicochemical parameters sampling and analysis methods}

In situ parameters such as, $\mathrm{pH}$, electrical conductivity (cond), temperature and dissolved oxygen $(\mathrm{O} 2)$ were measured with field multimeters (WTW 340i) before the macroinvertebrate sampling, and the velocity $(\mathrm{m} / \mathrm{s})$ with Global Water Flow Probe FP111. For the other parameters such as nutrients, 1.5 $\mathrm{L}$ of water was taken in a plastic bottle, stored on icefor further analysis in the laboratory. Nutrients was determined by molecular absorption spectrophotometry for Nitrate, Ortophosphate, Ammonium. All these parameters were measured with an accuracy ranking from 1 to $2 \%$ in the Laboratoire National d'Anlyse des Eaux which belong to the Ministère de l'Environment et du Development Durable [MEDD].

\section{Benthic macroinvertebrates sampling}

The benthic invertebrates were sampled with a hand net (rectangular opening: $25 \mathrm{~cm} \mathrm{x}$ $25 \mathrm{~cm}$, mesh size: $500 \mu \mathrm{m}$ ) and sorted following the same protocol as Kaboré et al. (2016). Multi-habitat sampling approach (Moog, 2007) was used, a pooled sample, consisting of 20 sampling units, was taken from all habitat types occupying a minimum of $5 \%$ or more of the study area. The number of sampling units allocated to each habitat type was proportional to the areal coverage of the latter. The habitats were composed of macrophytes (emerged and submerged plants), sediment (sand, mud, litter) and coarse substrate. The sampling started from downstream to upstream, the net was bumped against the bottom substrate to dislodge and collected organisms. The samples were kept in the alcohol (90\%) for detailed examination in the laboratory. Prior to sorting out the organisms, samples were sieved and the animals were separated. The animals were identified with the dichotomic microinvertebrates keys manuals of Tachet et al. (2003), Merrit et Cummins (1984), Lévêque et Durand (1981) and Moisan et Pelletier (2008) and with direct taxonomic expert support.

\section{Data analysis}

The total taxa richness (R) was simply taken as a count of number of species present in each site. We used Non-metric multidimentional scaling (NMS) scatter plot (presence-absence, distance measure: BrayCurtis similarity) of all sampling site and taxa to visualize possible predictors of faunal variation. The diversity indices provide more information about community composition, about scarcity and commonness of species in a given community; Shannon-Wiener index $(\mathrm{H})$, Equitability index are commonly used to characterize species diversity in a community. Shannon-Wiener diversity index was expressed following the equation [1] below, where pi is the proportion of individuals found in the ith taxon, $\mathrm{S}$ is the number of taxa in the samples.

$\mathbf{H}^{\prime}=-\sum_{\mathrm{i}=1}^{z} \mathrm{p}_{\mathrm{i}} \ln \mathrm{p}_{\mathrm{i}}$

Species equitability or evenness (E) was determined by the equation [2].

Equitability $(\mathrm{E})=\frac{\mathrm{H}}{\ln \mathrm{S}}$

Where;

$\mathrm{H}$ was the Shannon-Wiener index, $\mathrm{S}$ was the number of species in samples.

The density is an important tool to measure benthic community production in aquatic ecosystems (Barbour et al., 1996). The density was estimated following the equation [3]

$$
\mathrm{D}\left(\frac{\text { ind }}{\mathrm{m}^{2}}\right)=\frac{\text { Total Number of animals }}{\text { Area of samling units }}
$$

We conducted a Kruskal-Wallis ANOVA and Mann-Whitney tests with a software (SPSS, version 2.1) followed by pairwise comparison tests to compare taxa richness (R), ShannonWiener Diversity $(\mathrm{H})$, equitability (E) and biomass (D) between different rivers and 
seasons, and then between ecoregions. Finally, the canonical correspondence analysis (CCA) was performed to define composite environmental variables that correspond to the major patterns of community occurrence.

\section{RESULTS}

\section{Physicochemical parameters}

Table 1 summarizes the physical and chemical conditions of the study stations. Most of the sampled sites had warm waters (mean temperature of $29.9^{\circ} \mathrm{C}$ ). The $\mathrm{pH}$ values was slightly alkaline (mean $\mathrm{pH}$ of 7.24), with high conductivity $\left(\geq 100 \mu \mathrm{S} . \mathrm{cm}^{-1}\right)$. Some picks of variations in organic ions concentrations were observed between sampling sites.

\section{Benthic macroinvertebrates composition}

A total number of 33,357 specimens of benthic macroinvertebrates were collected. Eight orders of insect were recorded in the running water of the three rivers in Burkina Faso (Figure 2). All the sites were dominated by insects (relative abundance of 95\%), represented mostly $(80.3 \%)$ by midges and flies (Diptera). The mayflies (Ephemeroptera) and caddisflies (Trichoptera) made up 7.3\% of abundance. The Lepidoptera and Plecoptera, as well as the Bivalvia, Ostracoda and Arachnida were found in frequencies lower than $0.5 \%$.

\section{Taxa richness}

A high diversity of benthic macroinvertebrates with a total of 132 taxa was recorded in this study (Table 1). A large majority of these (103 taxa) belonged to 57 families from 8 orders of insects: Ephemeroptera, Odonata, Diptera, Coleoptera, Trichoptera, Lepidoptera, Plecoptera and Hemiptera. The remaining 27 taxa belonged to 11 families of Decapoda (Crustacea), Gastropoda and Bivalvia (Mollusca). Coleoptera represented the most diversified group of insects with the following families (taxa): Hydrophilidae (8), Elmidae (7), Dytiscidae (6) and the Noteridae (3). They were followed by Diptera (23), dominated by Chironomidae (4) and Simuliidae (4). Within the non-insect fauna we found an important diversity in Molluscs, with several species reported for Burkina Faso (Table 1). Thus, a total of 15 taxa of gastropod composed of Bulinidae (6), Thiaridae (3), Planorbidae (2), Ampullaridae (2), Lymnaeidae (1) and the Viviparidae (1) were observed. In the Bivalvia class, 3 families including seven species were recorded. Finally, we also found two species of freshwater shrimps belonging to the Palaemonidae and Atyidae families (Table A1).

\section{Seasonal and spatial variation on benthic macroinvertebrates community}

Figure 3 shows Non-metric multidimentional scaling analysis with spatial and temporal variation of all sampling sites. The three scatter plots did not reveal a clear difference between the three rivers (a), ecoregions (b), seasons (c) respectively based on the taxa occurrence (Figure 3). But, some distinct differences relative to the basins watersheds could be observed in the taxonomic composition (Figure 4 I). This mean overall taxonomic richness $(\mathrm{R}=19.63 \pm 3.77) \quad$ and Shannon-Wiener diversity index $(\mathrm{H}=2.00 \pm 0.33)$ were highest in Comoé and reached a minimum of $\mathrm{R}=8.30$ \pm 1.05 and $\mathrm{H}=1.12 \pm 0.12$ in Nakanbé River (Figure 4 I). While both Nakanbé (means of $\mathrm{R}=12.23 \pm 1.55, \quad \mathrm{D}=446.12 \pm 198.69, \quad \mathrm{E}=$ $0.63 \pm 0.05$ ) and Mouhoun (means of $\mathrm{R}=8.30$ $\pm 1.05, \quad \mathrm{D}=410.93 \pm 126.03, \quad \mathrm{E}=0.57 \pm 0.05)$ basins watersheds were distinguished by slightly high overall taxonomic richness, densities , Shannon-Wiener diversity and Equitability, respectively (Figure 4 I).

The overall taxonomic richness (mean of $\mathrm{R}=12.68 \pm 1.40$ ), Shannon-Wiener index (mean of $\mathrm{H}=1.51 \pm 0.15$ ), Equitability (mean of $\mathrm{E}=0.63 \pm 0.04$ ) and density (mean of $\mathrm{D}=$ $458.53 \pm 178.23$ ) tended to increase in Lower Soudanian eco-region compared to Upper Soudanian eco-region (mean of $\mathrm{R}=$ $10.19 \pm 1.53, \mathrm{H}=1.2 \pm 0.13, \mathrm{E}=0.6 \pm 0.04, \mathrm{D}=$ $343.12 \pm 106.68$, respectively see Figure 4 II).

In the Figure 4 III, the lowest density (mean of $\mathrm{D}=82.19 \pm 15.69$ ) was recorded in 
rainy season while the highest was recorded in dry season (mean of $\mathrm{D}=629.81$ ). In contrast, the high Shannon-Wiener index (mean of $\mathrm{H}=$ $1.6 \pm 0.20$ ), taxa richness (mean of $\mathrm{R}=$ $13.31 \pm 2.04$ ) and Equitability (mean of $\mathrm{E}=$ $0.64 \pm 0.06)$ were recorded in the rainy season. The taxa richness $(\mathrm{R}=10.31 \pm 1.37 ; 12.44 \pm$ 2.42), Shannon-Wiener index $(\mathrm{H}=1.29 \pm 014$; $1.39 \pm 0.16)$, Equitability $(\mathrm{E}=0.61 \pm 0.05 ; 0.59$ $\pm 0.05)$ did not reveal a big difference between the dry season and the end of rainy season, respectively $(p>0.05$, pairwise comparison tests, Figure 4 III e, f and g).

\section{Influence of physicochemical parameters on benthic macroinvertebrate assemblage}

Figure 5 shows the biplots of species

and physico-chemical parameters with eigenvalues for axis $1(0.36)$ and axis $2(0.23)$; both axis explained $59.4 \%$ of the variance of overall variables. The first two axes of CCA captured about $82.2 \%$ information of speciesenvironment correlations according to Monte Carlo test (pvalue $<0.05$ ). Thus, nitrate and orthophosphate $(\mathrm{r}=0.6$ all, respectively), ammonium $(\mathrm{r}=0.7)$ and the conductivity $(\mathrm{r}=0.8)$ are positively related to axis 1 . The Diptera including Chironomidae, Syrphidae, Culicidae, Psychodidae, and the Pulmonates molluscs frequency showed a strong correlation with the organic pollution; in contrast the Ephemeroptera, Plecoptera and Trichoptera are correlated with dissolved oxygen and water current.

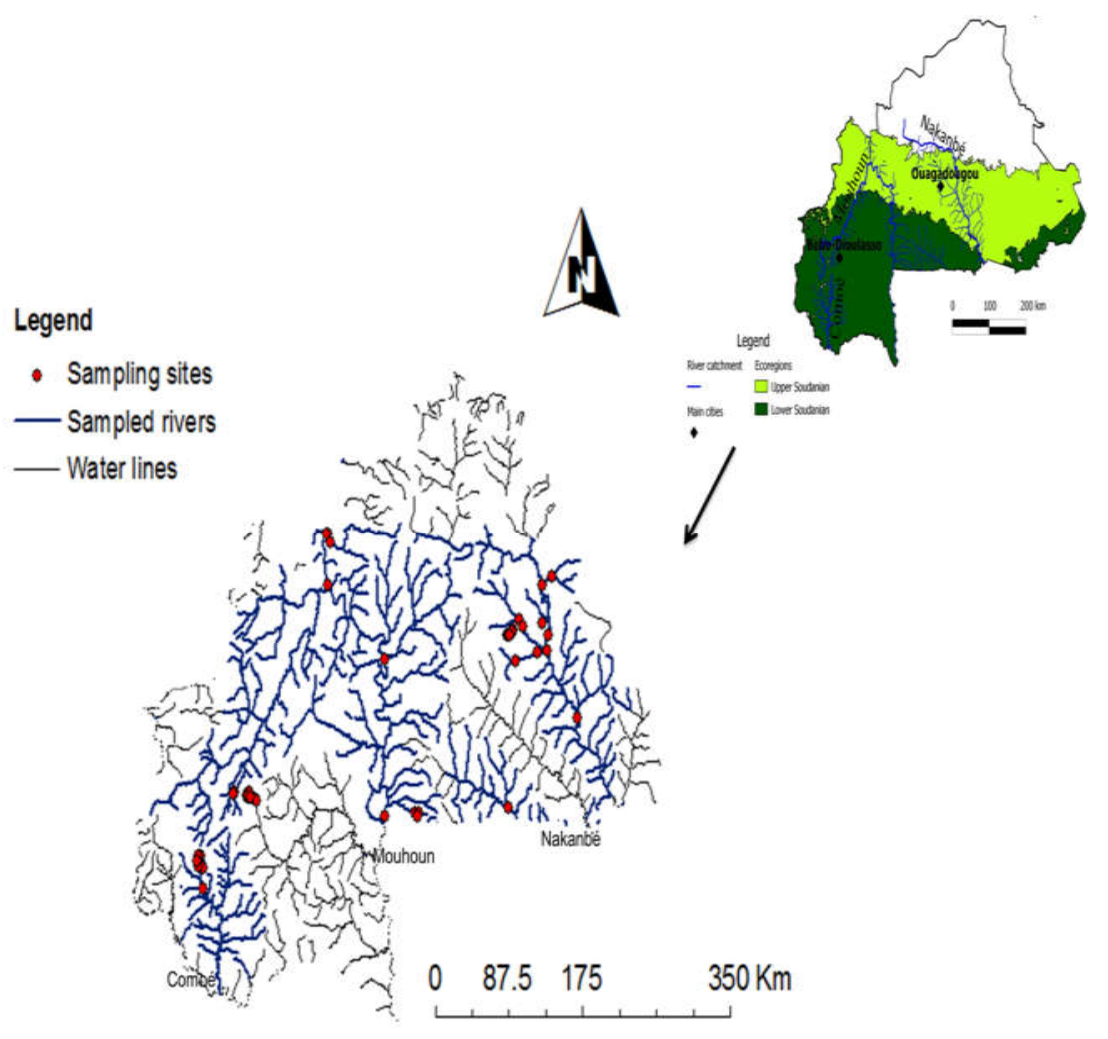

Figure 1: Map of study areas showing the Burkina Faso and the three rivers where the samples were taken. 

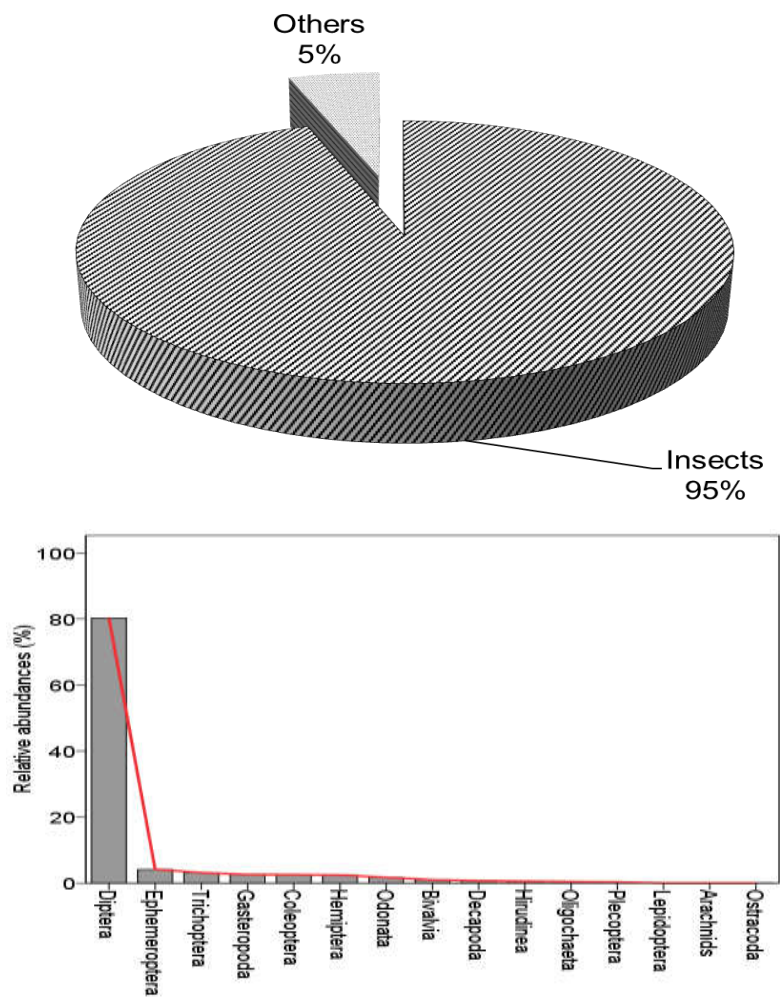

Figure 2: The relative abundances (\%) of benthic macroinvertebrate groups.
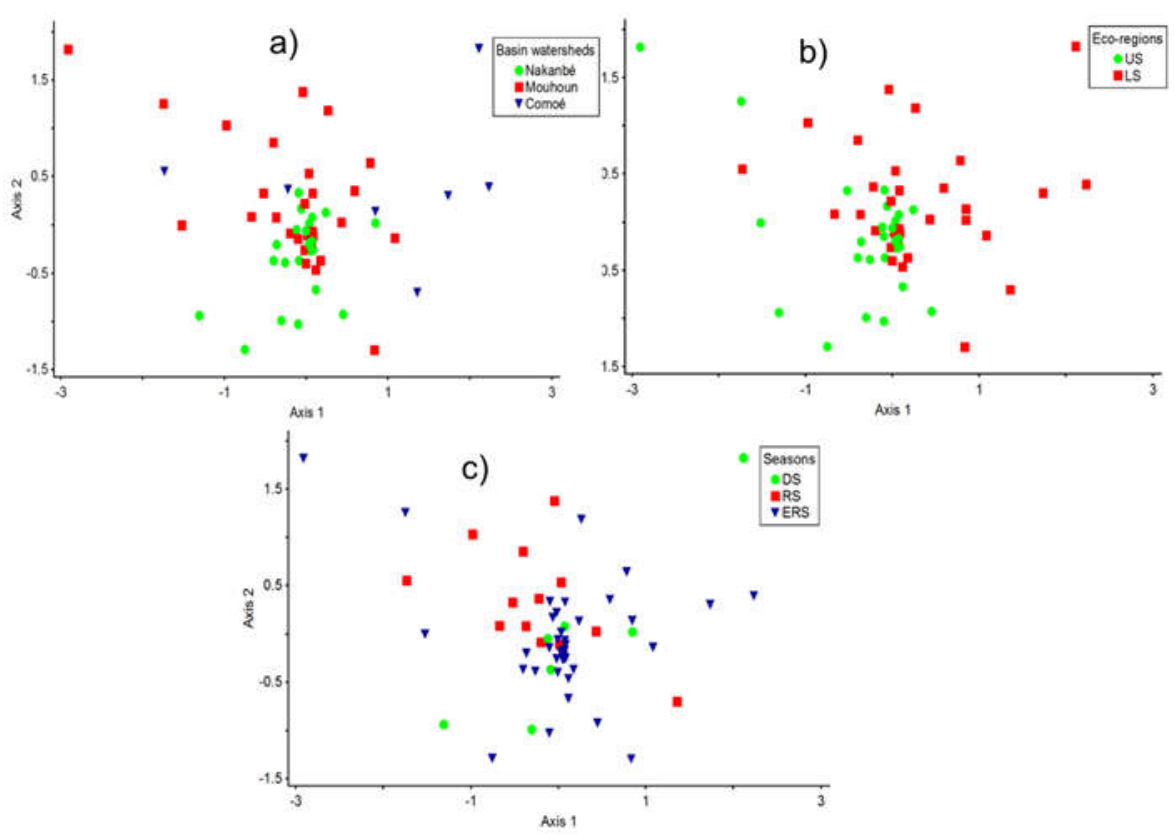

Figure 3: NMS-scatter plot of all samples $(\mathrm{N}=66)$ showing the spatial and temporal variation of benthic macroinvertebrates communities (Distance measure: Sorensen (Bray Curtis) using presence-absence transformed. $\mathrm{a})=$ indicates ecoregions, $\mathrm{b})=$ seasons and $\mathrm{c})=$ basins watersheds. $(17.57265=$ final stress for 2 -dimensional solution, $0.00000=$ final instability, $194=$ number of iterations). 


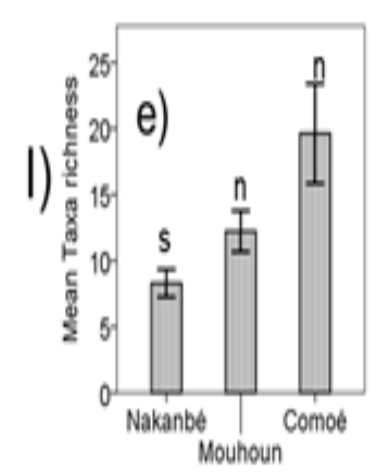

Basin watersheds
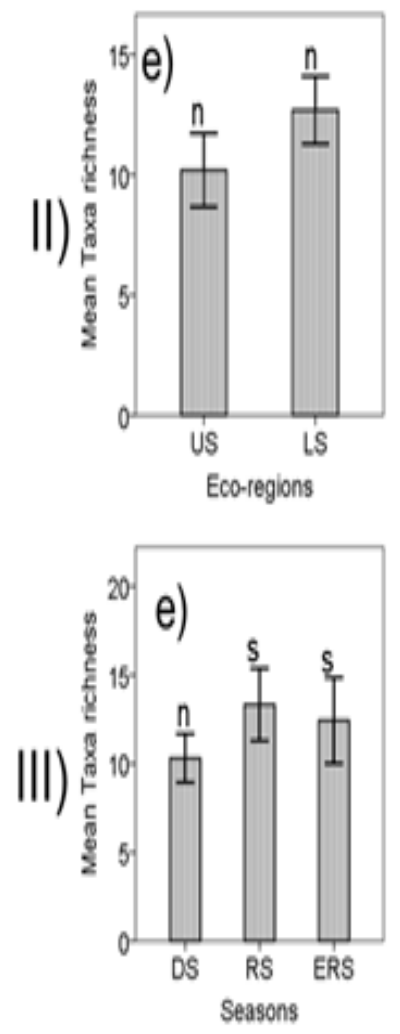

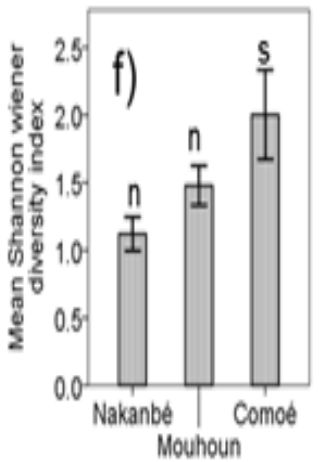

Basin watersheds

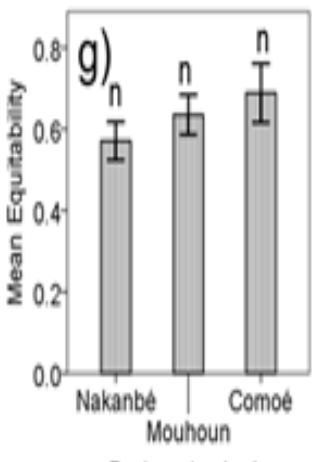

Basin watersheds
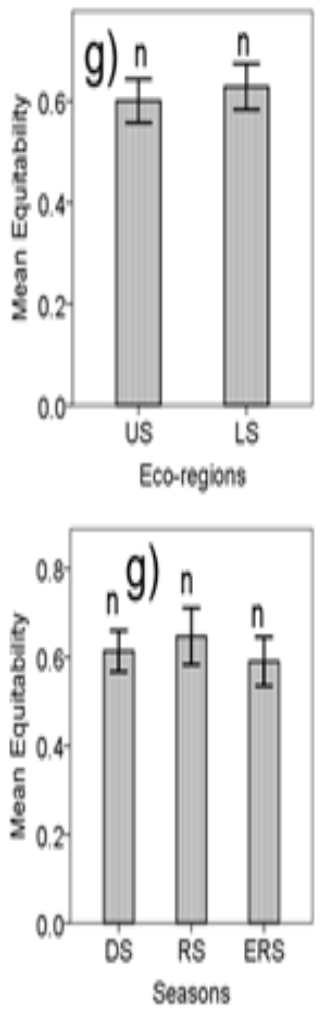

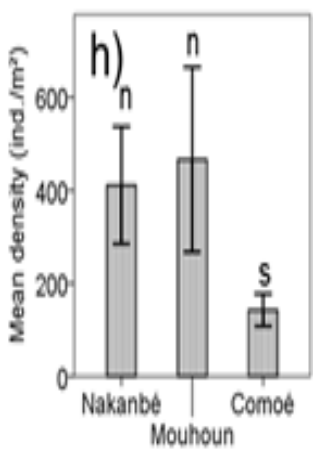

Basin watersheds
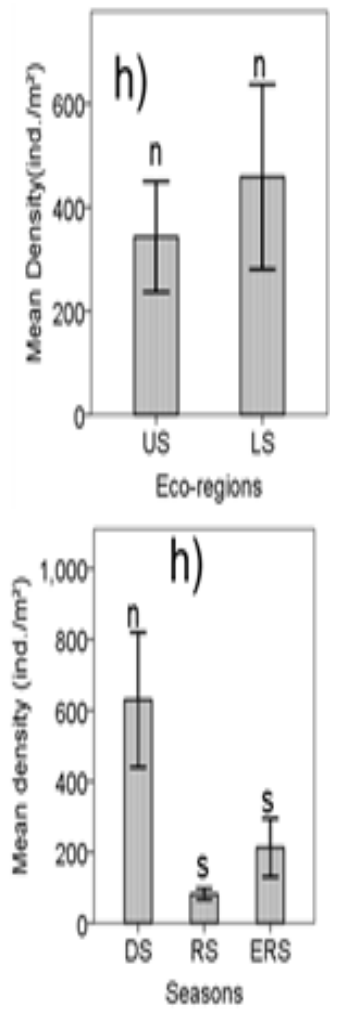

Figure 4: Diagram showing variation of taxa richness (e), Shannon-Wiener index (f), Equitability (g) and density (h) in different basin watersheds. Nakanbé, Mouhoun and Comoé (I), (II) R (e), H (f), E (g) and D (h) between different eco-regions. US: Upper Soudanian ecoregion, LS: Lower Soudanian ecoregion and III) R (e), H (f), E (g) and D (h) between different seasons. DS: dry season, RS: rainy season and ERS: end of rainy season. Letters above diagrams indicate statistical significance of differences between environmental factor types (pairwise comparison tests): only respective pairs with different alphabetical letters differ significantly $(\mathrm{P}<0.05)$. 


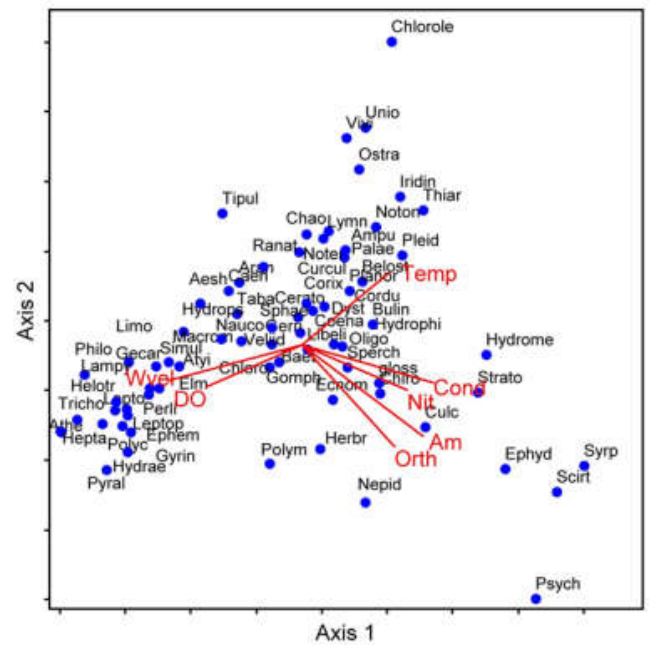

Figure 5: Relationship between physico-chemical variables and benthic macroinvertebrates based on canonical correspondance analysis $(\mathrm{CCA})$. Aran $=$ Araneae, Limo $=$ Limoniidae, Chiro=Chironomidae, Cerato $=$ Ceratopogonidae, Chao $=$ Chaoboridae, Culc $=$ Culicidae, Ephyd=Ephydridae, Tipul=Tipulidae, Strato=Stratomyiidae, Syrp=Syrphidae, Taba $=$ Tabanidae, Athe=Athericidae, Simul=Simuliidae, Psych=Psychodidae, Gecar=Gecarcinucidae, Atyi=Atyidae, Ostra $=$ Ostracoda, Palae=Palaemonidae $\quad$ Caen=Caenidae $\quad$ Bae=Baetidae, Ephem=Ephemerelliidae, Hepta=Heptageniidae, Leptop=Leptophlebiidae, Tricho=Trichorythidae, Polym=Polymitarcyidae, Ecnom=Ecnomidae, Hydrops=Hydropsychidae, Lepto=Leptoceridae, Philo=Philopotamidae, Polyc=Polycentropodidae, Gomph=Gomphidae, Aesh= Aeshnidae, Chloro=Chlorocyphidae, Chlorole $=$ Chlorolestidae, Coena=Coenagrionidae, Cordu=Cordullidae, Libeli=Libelilludae, Macrom=Macromiidae, Curcul=Curculionidae, Dyst=Dytiscidae, Elm=Elmidae, Lampy=Lampyridae, Hydrophi=Hydrophilidae, Hydrae=Hydraenidae, Gyrin=Gyrinidae, Noter=Noteridae, Sperch=Spercheidae, Scirt=Scirtidae, Corix $=$ Corixidae, Gerri $=$ Gerridae, Helotr $=$ Helotrephidae, Herbr=Herbridae, Hydrome=Hydrometridae, Belost=Belostomatidae, Nauco=Naucoridae, Nepid=Nepidae, Noton=Notonectidae, Veliid=Veliidae, Pleid=Pleidae, Ranat=Ranatridae, Bulin=Bulinidae, Lymn=Lymnaeidae, Planor=Planorbidae, Ampu=Ampullaridae, Thiar=Thiaridae, gloss=glossiphoniidae, Oligo $=$ Oligochaeta, Perli=Perlidae, Pyral=Pyralidae, Iridin=Iridinidae, Sphae=Sphaeriidae, Unio=Unionidae, Vivi=Viviparidae.

Table 1: Summary of physicochemical parameters of study sites in Burkina Faso water bodies

\begin{tabular}{lll}
\hline Water variables & Rivers & Mean \\
\hline \multirow{3}{*}{ Temperature $\left({ }^{\circ} \mathrm{C}\right)[\mathrm{Tem}]$} & Comoé & $26.92( \pm 0.46)$ \\
& Mouhoun & $30.55( \pm 0.42)$ \\
& Nakanbé & $29.95( \pm 0.65)$ \\
$\mathrm{PH}$ & Comoé & $7.94( \pm 0.27)$ \\
& Mouhoun & $6.97( \pm 0.11)$ \\
& Nakanbé & $7.33( \pm 0.12)$ \\
Conductivity $(\mu \mathrm{S} / \mathrm{cm})[\mathrm{Cond}]$ & Comoé & $57.97( \pm 15.56)$ \\
& Mouhoun & $167.80( \pm 24.05)$ \\
& Nakanbé & $440.71( \pm 79.54)$ \\
& Comoé & $6.47( \pm 0.85)$ \\
Dissolved Oxygen $(\mathrm{mg} / \mathrm{l})[\mathrm{DO}]$ & Mouhoun & $4.36( \pm 0.43)$ \\
& Nakanbé & $5.16( \pm 0.62)$ \\
Nitrate $(\mathrm{mg} / \mathrm{l})[\mathrm{Nit}]$ & Comoé & $0.74( \pm 0.07)$ \\
& Mouhoun & $5.90( \pm 1.72)$ \\
& Nakanbé & $12.20( \pm 3.07)$ \\
\multirow{2}{*}{ Ortophosphate $(\mathrm{mg} / \mathrm{l})[\mathrm{Orth}]$} & Comoé & $0.26( \pm 0.05)$ \\
\multirow{2}{*}{ Ammonium $(\mathrm{mg} / \mathrm{l})[$ Amm $]$} & Mouhoun & $2.31( \pm 0.58)$ \\
& Nakanbé & $2.83( \pm 0.65)$ \\
& Comoé & $1.52( \pm 0.85)$ \\
\multirow{2}{*}{ Water velocity $(\mathrm{m} / \mathrm{s})[\mathrm{Wvel}]$} & Mouhoun & $2.05( \pm 0.45)$ \\
& Nakanbé & $5.19( \pm 1.86)$ \\
& Comoé & $0.68( \pm 0.12)$ \\
& Mouhoun & $0.32( \pm 0.07)$ \\
& Nakanbé & $0.08( \pm 0.03)$ \\
\hline
\end{tabular}


Table A1: List of the aquatic benthic macroinvertebrates taxa recorded in this study.

\begin{tabular}{|c|c|c|c|c|c|c|c|}
\hline Embranchement & Classes & Orders & Families & Taxa & Abundances & Densities (ind $/ \mathbf{m}^{2}$ ) & Frequencies \\
\hline & \multirow{8}{*}{ Insecta } & \multirow{8}{*}{ Coleoptera } & Curculionidae & Stenolophus angolensis & 4 & 3.2 & 4.55 \\
\hline & & & \multirow{6}{*}{ Dytiscidae } & Bidessini sp. & 10 & 8 & 3.03 \\
\hline & & & & Hydrovatus sp. & 136 & 108.8 & 21.21 \\
\hline & & & & Methles sp. & 9 & 7.2 & 4.55 \\
\hline & & & & Laccophilus sp. & 41 & 32.8 & 15.15 \\
\hline & & & & Neptosternus sp. & 142 & 113.6 & 7.58 \\
\hline & & & & Yola sp. & 9 & 7.2 & 4.55 \\
\hline & & & Elmidae & Dubiraphia sp. & 16 & 12.8 & 7.58 \\
\hline
\end{tabular}

Table A1. (Continued)

\begin{tabular}{|c|c|c|c|c|c|c|c|}
\hline Embranchement & Classes & Orders & Families & Taxa & Abundances & Densities (ind $/ \mathbf{m}^{2}$ ) & Frequencies \\
\hline & & & & Elmis sp. & 20 & 16 & 3.03 \\
\hline & & & & Stenelmis sp. & 14 & 11.2 & 6.06 \\
\hline & & & & Microdinodes sp. & 3 & 2.4 & 3.03 \\
\hline & & & & Leptelmis sp. & 8 & 6.4 & 4.55 \\
\hline & & & & Potamodytes sp. & 7 & 5.6 & 3.03 \\
\hline & & & & Pseudomacronychus sp. & 73 & 58.4 & 15.15 \\
\hline & & & Gyrinidae & Orectogyrus sp. & 16 & 12.8 & 3.03 \\
\hline & & & Hydraenidae & Hydraenopsis sp. & 6 & 4.8 & 3.03 \\
\hline & & & & Amphios sp. & 28 & 22.4 & 15.15 \\
\hline & & & Hydrophilidae & Berosus sp. & 26 & 20.8 & 7.58 \\
\hline
\end{tabular}


Table A1. (Continued)

\begin{tabular}{|c|c|c|c|c|c|c|c|}
\hline Embranchement & Classes & Orders & Families & Taxa & Abundances & Densities (ind./m²) & Frequencies \\
\hline & & & & Enochrus sp. & 20 & 16 & 7.58 \\
\hline & & & & Sternolophus sp. & 36 & 28.8 & 4.55 \\
\hline & & & & Helochares sp. & 49 & 39.2 & 19.70 \\
\hline & & & & Helocharimorphus sp. & 4 & 3.2 & 1.52 \\
\hline & & & & Ceolostoma sp. & 6 & 4.8 & 6.06 \\
\hline & & & & Regimbartia sp. & 11 & 8.8 & 6.06 \\
\hline & & & Lampyridae & Lampyridae & 2 & 1.6 & 3.03 \\
\hline & & & & Hydrocanthus sp. & 20 & 16 & 7.58 \\
\hline & & & Noteridae & Neohydrochantus sp. & 12 & 9.6 & 3.03 \\
\hline & & & & Canthydrus sp. & 79 & 63.2 & 15.15 \\
\hline & & & Scirtidae & Scirtidae & 30 & 24 & 3.03 \\
\hline
\end{tabular}

Table A1. (Continued)

\begin{tabular}{|c|c|c|c|c|c|c|c|}
\hline Embranchement & Classes & Orders & Families & Taxa & Abundances & Densities (ind./m²) & Frequencies \\
\hline & & \multirow{11}{*}{ Diptera } & Spercheidae & Spercheus sp. & 21 & 16.8 & 4.55 \\
\hline & & & Athericidae & Atherix sp. & 6 & 4.8 & 3.03 \\
\hline & & & Ceratopogonidae & Bezzia sp. & 28 & 22.4 & 15.15 \\
\hline & & & Chaoboridae & Chaoborus sp. & 14 & 11.2 & 10.61 \\
\hline & & & \multirow{4}{*}{ Chironomidae } & Chironomini sp. & 4097 & 3277.6 & 43.94 \\
\hline & & & & Chironomus sp. & 15468 & 12374.4 & 33.33 \\
\hline & & & & Tanypus sp. & 126 & 100.8 & 25.76 \\
\hline & & & & Tanytarsus sp. & 37 & 29.6 & 9.09 \\
\hline & & & \multirow{2}{*}{ Culicidae } & Culicidae & 3208 & 2566.4 & 25.76 \\
\hline & & & & Aedes sp. & 159 & 127.2 & 10.61 \\
\hline & & & Ephydridae & Ephydridae & 174 & 139.2 & 4.55 \\
\hline
\end{tabular}


Table A1. (Continued)

\begin{tabular}{|c|c|c|c|c|c|c|c|}
\hline Embranchement & Classes & Orders & Families & Taxa & Abundances & Densities (ind./ $\mathbf{m}^{2}$ ) & Frequencies \\
\hline & & & Limoniidae & Antocha sp. & 1 & 0.8 & 1.52 \\
\hline & & & Macromiidae & Macromia sp. & 3 & 2.4 & 3.03 \\
\hline & & & Psychodidae & Psychodidae & 1508 & 1206.4 & 13.64 \\
\hline & & & & Simulium sp. & 1516 & 1212.8 & 18.18 \\
\hline & & & Simuliido & Simulium aureosimile & 2 & 1.6 & 1.52 \\
\hline & & & simumidae & Simulium ruficorne & 7 & 5.6 & 1.52 \\
\hline & & & & Simulium adersi & 6 & 4.8 & 1.52 \\
\hline & & & Stratomyiidae & Stratomyiidae & 4 & 3.2 & 4.55 \\
\hline & & & Surnhidge & Chrysogaster sp. & 68 & 54.4 & 1.52 \\
\hline & & & syrpnidae & Eristalis sp. & 314 & 251.2 & 13.64 \\
\hline & & & Tohonidop & Chrysops sp. & 19 & 15.2 & 13.64 \\
\hline & & & I abanidae & Tabanus sp. & 4 & 3.2 & 3.03 \\
\hline
\end{tabular}

Table A1. (Continued)

\begin{tabular}{|c|c|c|c|c|c|c|c|}
\hline Embranchement & Classes & Orders & Families & Taxa & Abundances & Densities (ind./m²) & Frequencies \\
\hline & & \multirow{11}{*}{ Hemiptera } & \multirow[t]{2}{*}{ Tipulidae } & Tipulidae & 5 & 4 & 3.03 \\
\hline & & & & Diplonycus sp. & 30 & 24 & 16.67 \\
\hline & & & \multirow{2}{*}{ Belostomatidae } & Appasus sp. & 92 & 73.6 & 3.03 \\
\hline & & & & Belostoma sp. & 37 & 29.6 & 9.09 \\
\hline & & & \multirow[t]{2}{*}{ Corixidae } & Micronecta sp. & 255 & 204 & 24.24 \\
\hline & & & & Limnogonus sp. & 4 & 3.2 & 6.06 \\
\hline & & & \multirow[t]{2}{*}{ Gerridae } & Hynesionella sp. & 10 & 8 & 3.03 \\
\hline & & & & Rhagadotarsus sp. & 22 & 17.6 & 16.67 \\
\hline & & & Nepidae & Laccotrephes sp. & 12 & 9.6 & 3.03 \\
\hline & & & Ranatridae & Ranatra sp. & 7 & 5.6 & 7.58 \\
\hline & & & Notonectidae & Notonecta sp. & 106 & 84.8 & 13.64 \\
\hline
\end{tabular}


Table A1. (Continued)

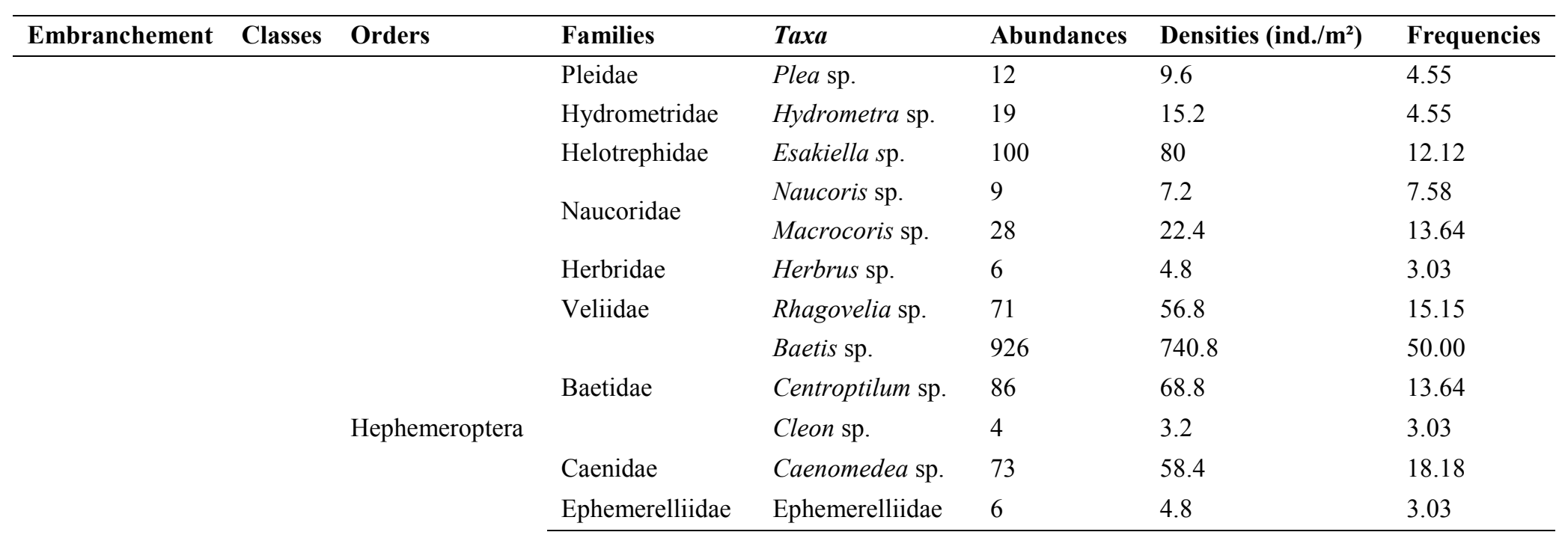

Table A1. (Continued)

\begin{tabular}{|c|c|c|c|c|c|c|c|}
\hline Embranchement & Classes & Orders & Families & Taxa & Abundances & Densities (ind./m²) & Frequencies \\
\hline & & & Heptageniidae & Notonurus sp. & 61 & 48.8 & 7.58 \\
\hline & & & Leptophlebiidae & Euthraulus sp. & 204 & 163.2 & 12.12 \\
\hline & & & Polymitarcyidae & Ephoron sp. & 18 & 14.4 & 6.06 \\
\hline & & & Tricorythidae & Trichorythus sp. & 15 & 12 & 3.03 \\
\hline & & Lepidoptera & Pyralidae & Pyralidae & 7 & 5.6 & 4.55 \\
\hline
\end{tabular}


I. KABORÉ et al. / Int. J. Biol. Chem. Sci. 10(4): 1542-1561, 2016

\begin{tabular}{llllll} 
& Aeshnidae & Anax sp. & 2 & 1.6 & 3.03 \\
\cline { 3 - 4 } Odonata & Chlorocyphidae & Chlorocypha sp. & 21 & 16.8 & 6.06 \\
& & Coenagrion sp. & 108 & 86.4 & 25.76 \\
& \multirow{3}{*}{ Coenagrionidae } & Enallagma sp. & 20 & 16 & 1.52 \\
& & Ishnura sp. & 62 & 49.6 & 6.06 \\
& Pseudagrion sp. & 124 & 99.2 & 13.64 \\
& Corduliidae & Phyllomacromia sp. & 19 & 15.2 & 10.61 \\
\hline
\end{tabular}

Table A1. (Continued)

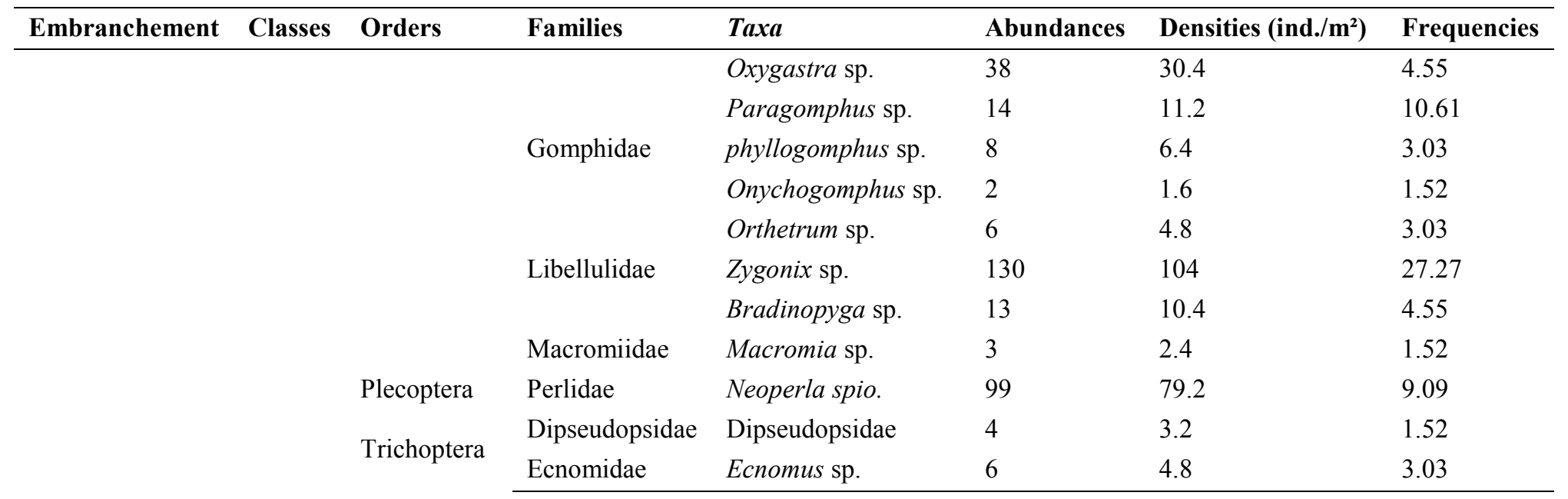


Table A1. (Continued)

\begin{tabular}{|c|c|c|c|c|c|c|c|}
\hline Embranchement & Classes & Orders & Families & Taxa & Abundances & Densities (ind./m²) & Frequencies \\
\hline & & & \multirow{2}{*}{ Hydropsychidae } & Cheumatopsyche digitate & 878 & 702.4 & 16.67 \\
\hline & & & & Macronematini & 2 & 1.6 & 1.52 \\
\hline & & & Leptoceridae & Leptocerus sp. & 69 & 55.2 & 10.61 \\
\hline & & & Philopotamidae & Chimarra petri & 51 & 40.8 & 3.03 \\
\hline & Malacostraca & Decapoda & Atyidae & Caridina africana & 135 & 108 & 13.64 \\
\hline & Ostracoda & & & Ostracoda & 4 & 3.2 & 6.06 \\
\hline \multirow{2}{*}{ Mollusca } & \multirow{2}{*}{ Bivalvia } & \multirow{2}{*}{ Unionoida } & \multirow{2}{*}{ Iridinidae } & Aspatharia sp. & 7 & 5.6 & 1.52 \\
\hline & & & & Chambardia wahbergi & 4 & 3.2 & 1.52 \\
\hline
\end{tabular}

Table A1. (Continued)

\begin{tabular}{|c|c|c|c|c|c|c|c|}
\hline Embranchement & Classes & Orders & Families & Taxa & Abundances & Densities (ind./m²) & Frequencies \\
\hline & & & \multirow{4}{*}{ Unionidae } & Mutela rostrata & 14 & 11.2 & 6.06 \\
\hline & & & & Mutela sp. & 5 & 4 & 1.52 \\
\hline & & & & Coelatura aegyptiaca & 2 & 1.6 & 1.52 \\
\hline & & & & Coelatura sp. & 279 & 223.2 & 4.55 \\
\hline & & Veneroida & Sphaeridae & Sphaerium sp. & 17 & 13.6 & 12.12 \\
\hline & Gastropoda & Architaenioglossa & Ampullaridae & Lanistes ovum & 10 & 8 & 3.03 \\
\hline
\end{tabular}


I. KABORÉ et al. / Int. J. Biol. Chem. Sci. 10(4): 1542-1561, 2016

$\begin{array}{lllll} & \text { Lanistes varicus } & 23 & 18.4 & 10.61 \\ \text { Lymneidae } & \text { Lymnae natalensis } & 46 & 36.8 & 10.61 \\ & \text { Biomphalaria sp } & 9 & 7.2 & 6.06 \\ \text { Planorbidae } & \text { Biomphalaria pfeifferi } & 172 & 137.6 & 13.64 \\ & \text { Bulinus camerunensis } & 30 & 24 & 4.55 \\ \text { Bulinidae } & \text { Bulinus troncatus } & 2 & 1.6 & 1.52\end{array}$

Table A1. (Continued)

\begin{tabular}{|c|c|c|c|c|c|c|c|}
\hline Embranchement & Classes & Orders & Families & Taxa & Abundances & Densities (ind./ $\mathbf{m}^{2}$ ) & Frequencies \\
\hline & & & & Bulinus forskali & 6 & 4.8 & 4.55 \\
\hline & & & & Bulinus globosus & 5 & 4 & 4.55 \\
\hline & & & & Bulinus jousseaumei & 23 & 18.4 & 3.03 \\
\hline & & & & Bulinus senegalensis & 44 & 35.2 & 12.12 \\
\hline & & & Viviparidae & Bellamya unicolor & 48 & 38.4 & 4.55 \\
\hline & & & & Cleopatra bulimoïdes & 233 & 186.4 & 9.09 \\
\hline & & Sorbeoconcha & Thiaridae & Cleopatra sp. & 212 & 169.6 & 6.06 \\
\hline \multirow{4}{*}{ Annelida } & & & & Potamoda sp. & 1 & 0.8 & 1.52 \\
\hline & \multirow{2}{*}{ Clitellata } & & & Hirudinea & 182 & 145.6 & 10.61 \\
\hline & & & & Oligochaeta & 149 & 119.2 & 28.79 \\
\hline & Arachnid & & & Arachnid & 6 & 4.8 & 6.06 \\
\hline
\end{tabular}




\section{DISCUSSION}

Freshwater benthic macroinvertebrates was characterized by the Arthropoda $=>$ Mollusca $=>$ Annelida (Akindele and Liadi, 2014; Tampo et al., 2015), as confirmed by this study. This taxonomic list recorded is common to the traditional one reported in freshwaters of Burkina Faso (Guenda, 1996; Sanogo, 2014) and other Afrotropical regions (Edia et al., 2013; Kouadio et al., 2008; Okorafor et al., 2012). Compared to the others studies in some West Africa rivers, Burkina Faso streams appears rich in benthic macroinvertebrates. These results are similar to those reported by Vinson and Hawkins (1996), Camara et al. (2012), Kearns and Stevenson (2012) and Kaboré et al. (2016) who have demonstrated that the sampling technique employed and the types of habitats (e.g. natural habitats, multi-habitats) can explain the high number of taxa found in a given study. The benthic macroinvetrebrates composition presents a strong seasonal and spatial effects variation which can be justified by the variation of water quantity and mouvement. The year can be divided into three sub-hydrological periods to characterize the benthic maxroinvertebrates structure. Although we recorded high number of taxa and the lowest biomass in the rainy season. This may be explained by the fact that the runoff brings a great quantity of foods consisted of organic matter, bacteria, phytoplakton and Zooplankton (Ouéda et al., 2007) into the water bodies; which improve food condition for benthic macroinvertebrates. In Addition, the high taxa richness could also be attributed to abundance of macrophytes that enhance environmental heterogeneity, provide protection from predators and reduced competition betweeen species (Uwadiae, 2013; Gong et al., 2000; Kaboré et al., 2016). However the lowest density at the same time is due to enlargement of ecological niche (e.g. expanding of flooded areas). Comparing the three basin watersheds benthic macroinvertebrates, we found that Nakanbé bears the low taxa richness. The similar trend was observed in Upper Soudanian ecoregion mainly drained by Nakanbé river. Melcher et al. (2012) and Ouedraogo (2010) have demonstrated that Nakanbé basin watershed is the most populated and dammed area with intensly anthropogenic activities and urbanisation; as a consequence, these pressures deteriorate the water quality and the river systems and finally lead to multiple biodiversity extinctions. The poor water quality could be attributed to several maninduced activities such as urban runoff to surface river water, the waste dumps into the rivers (Wright et al., 1995; Aggrey-Fynn et al., 2011; Kaboré et al., 2016). The benthic macroinvetebrates may have different levels of sensitivity to pollution and many abiotic factors in the river ecosystems. The high values of conductivity, nitrate, ammonium , orthophoshate and the low values of dissolved oxygen observed in somes sites are indications of deteriorated water quality as a result of various anthropogenic activities in that sites (Amiro et al., 2010; Tampo et al., 2015). Such anthropogenic pollution corroborates with the use of agricultural fertilizers and urban sewage both pollutants are believed to increase the concentrations of organic ions in the water bodies (Sanogo and Kabré, 2014; Ouedraogo et al., 2015). Ouéda et al. (2007) and Kaboré et al. (2016) observed similar trend of organic ions in density inhabited area and urban streams where wastes (e.g. domestic, industrials and urban) are constantly discharged into the stream/rivers. Which, should justify the relationship between these variables with tolerant taxa such as Syrphidae, Culicidae, Psychodidae, as well as the Pulmonates molluscs that increase with increasing disturbance (Nkwoji et al., 2010; Moya et al., 2012; Tampo et al., 2015). Through the sensitive taxa Ephemeroptera (Heptageniidae, Ephemerelliidae), Plecoptera and Trichoptera taxa are often the most abundant insects encountered in sites with a sufficiently high dissolved oxygen concentration and good 
habitats condition (Arimoro et al., 2010; Shelly et al., 2011).

\section{Conclusion}

This study described the benthic macroinvertebrates community dynamic in three main rivers in Burkina Faso. We recorded high taxa richness, indicating that the streams/rivers of Burkina Faso appear very rich. Among the factors potentially important that explain the possible predictors of faunal relationships, we observed some differences in community structure suggesting that environmental factors such as the seasons, the activities in basins watershed and the physicochemical variables could play a key role in the distribution pattern of benthic macroinvertebrates in Burkina Faso. In further study, it is allowed, from this present work to address on the one hand the nutritional role of each available habitats and physicochemical factors on the aquatic communities and on the other hand the role of the aquatic macroinvertebrates as biological indicators of detoriated habitats.

\section{COMPETING INTERESTS}

The authors declare that they have no competing interests.

\section{AUTHORS' CONTRIBUTIONS}

IK: Conception, field work design, manuscript writing, systematic, data processing. IO: Field work, systematic, manuscript reading and editing. LT: Manuscript review and editing, data processing. AO: Study design, data processing, manuscript review. OM: Field work design, manuscript review and editing, supervision. WG: Systematic and Supervision. AHM: Supervision, manuscript review and editing, project leader.

\section{ACKNOWLEGEMENTS}

We are grateful to APPEAR programme (Austrian Partnership Programme in Higher Education and Research for Development), funded by the ADA (Austrian
Development Agency) and implemented by the OEAD (Austrian Agency for International Cooperation in Education and Research) for funding this study. We thank two anonymous reviewers for many insightful comments and suggestions which largely helped to improve the manuscript. Additionally, we thank Mr. THIEMOUNOU Daouda for comments and English proof reading.

\section{REFERENCES}

Aggrey-Fynn J, Galyuon I., Aheto D, Okyere I. 2011. Assessment of the environmental conditions and benthic macroinvertebrate communities in two coastal lagoons in Ghana. Ann. Biol. Res., 2(5): 413-424.

Akindele EO, Liadi AA. 2014. Diversity and response of benthic macroinvertebrates to Natural and Induced Environmental Stresses in Aiba Stream, Iwo, Southwestern Nigeria. West Afr. J. Appl. Ecol., 22(1): 101-111.

Arimoro FO, Muller WJ. 2010. Mayfly (Insecta: Ephemeroptera) community structure as an indicator of the ecological status of a stream in the Niger Delta area of Nigeria. Environ. Monit. Assess., 166 (1-4): $\quad 581-594 . \quad$ DOI: http://dx.doi.org/10.1007/s10661-0091025-3

Barbour MT, Gerritsen J, Snyder BD, Stribling JB. 1999. Rapid Bioassessment Protocols for Use in streams and Wadeable Rivers: Periphyton, Benthic Macroinvertebrates and Fish, Second Edition. EPA 841-B-99-002. U.S. Environmental Protection Agency; Office of Water; Washington, D. C.

Barbour MT, Gerritsen J, Griffith GE, Frydenborg R, McCarron E, White JS, Bastian ML. 1996. A framework for biological criteria for Florida streams using benthic macroinvertebrates. $J$. North. Am. Benthol. Soc., 15: 185-211. DOI: http://dx.doi.org/10.2307/1467948

Barry B, Obuobie E, Andreini M, Andah W, Pluquet M. 2005. The Volta river basin Comprehensive assessment of water 
management in agriculture. Comparative study of river basin development and management. $198 \mathrm{p}$ Available online http://www.iwmi.cgiar.org/Assessment/R esearch_Projects/River_Basin_Developm ent_and_Management/Projects_Locations /volta_river_burkina_faso_ghana.htm.

Camara IA, Diomande D, Bony YK, Ouattara A, Franquet E, Gourene G. 2012. Diversity assessment of benthic macroinvertebrate communities in Banco National Park (Banco Stream , Côte d'Ivoire), Afr. J. Ecol., 50: 205-217. DOI: http://dx.doi.org/10.1111/j.13652028.2011.01312.x

Covich AP, Palmer MA, Crowl TA. 1999. The role of benthic invertebrate species in freshwater ecosystems: zoobenthic species influence energy flows and nutrient cycling. BioScience, 49(2): 119127. DOI: http://dx.doi.org/10.2307/1313537

Edia OE, Bony KY, Konan KF, Ouattara A, Gourène G. 2013. Distribution of Aquatic Insects among Four Costal River Habitats. Bull. Env. Pharmacol. Life Sci., 2: 68-77.

George ADI, Abowei JFN, Alfred-Ockiya JF. 2010. The Distribution, Abundance and Seasonality of Benthic Macro Invertebrate in Okpoka Creek Sediments, Niger Delta, Nigeria. Res. J. Appl. Sci. Eng. Tech., 2(1): 11-18.

Gong Z, Xie P, Wang S. 2000. Macrozoobenthos in 2 shallow, mesotrophic Chinese lakes with contrasting sources of primary production. J. North. Am. Benthol. Soc., 19(4): 709-724. DOI : http://dx.doi.org/10.2307/1468128

Guenda W. 1985. Evaluation de la toxicité de l'Abate sur les macroinvertébrés de la Volta Rouge (Burkina Faso-Ghana), dans le cadre du contrôle de l'onchocercose en Afrique Occidentale. Bull. I.F.A.N: T.46, sér. A nº3-4, 1986-1987.

Guenda W. 1996. Etude faunistique, écologique et de la distribution des insects d'un réseau hydrographique de l'Ouest africain: le Mouhoun (Burkina Faso) ; rapport avec Similium damnosum Theobald, vecteur de l'onchocercose. Thèse d'état, Université Aix-Marseille, France, p. 260.

Kaboré I, Moog O, Alp M, Guenda W, Koblinger T, Mano K, Ouéda A, Ouédraogo R, Trauner D, Melcher AH. 2016. Using macroinvertebrates for ecosystem health assessment in semi-arid streams of Burkina Faso. Hydrobiologia 766(1): 57-74. DOI: http://dx.doi.org/10.1007/s10750-0152443-6

Kearns P, and Stevenson RD. 2012. The Effect of Decreasing Temperature on Arthropod Diversity and Abundance in Horse Dung Decomposition Communities of Southeastern Massachusetts. Psyche, 2012: 1-12. DOI: http://dx.doi.org/10.1155/2012/618701

Koblinger T, Trauner D. 2013. Benthic invertebrate assemblages in water bodies of Burkina Faso. Master Thesis ,University of Natural Resources and Life Sciences, Vienna, Austria, p. 147.

Kouadio KN, Diomandé D, Ouatara A, Kone YJM, Gourene G. 2008. Distribution of benthic macroinvertebrate communities in relation to environmental factors in the ebrie lagoon (Ivory Coast, West Africa). Pakistan J. Biol. Sci., 61(2): 59-69.

Lévêque C, Durand JR. 1981. Flore et Faune aquatiques de l'Afrique SaheloSoudanienne (Tome II). IRD Editions.

Marzin A. 2013. Ecological assessment of running water using bio-indicator: associated variability and uncertainty. Doctorate thesis, Agros Paris Tech, Paris, p. 202.

Melcher AH, Ouedraogo R, Schmutz S. 2012. Spatial and seasonal fish community patterns in impacted and protected semiarid rivers of Burkina Faso. Ecol. Eng., 48, 117-129. DOI: http://dx.doi.org/10.1016/j.ecoleng.2011. 07.012 
Merrit RW, Cummins KW. 1984. An Introduction to the Aquatic Insects of North America (2nd edn). Kendall/Hunt Publishing Company; Dubuque (Iowa); 441.

Mesa LM, Reynaga MC, Correa MV, Sirombra MG. 2013. Effects of anthropogenic impacts on benthic macroinvertebrates assemblages in subtropical mountain streams. Iheringia, Porto Alegre, Sér. Zool., 103 (4): 342349.

DOI:

http://dx.doi.org/10.1590/S0073-

47212013000400002

Moisan J, Pelletier L. 2008. Guide de surveillance biologique basée sur les macroinvertébrés benthiques d'eau douce du Québec - Cours d'eau peu profonds à substrat grossier. Direction du suivi de l'état de l'environnement, Ministère du Développement durable, de l'Environnement et des Parcs, Québec, Canada, p. 86.

Moore AA, Palmer MA. 2005. Invertebrate biodiversity in agricultural and urban headwater streams: implications for conservation and management. Ecol. Appl., 15(4): 1169-1177. DOI: http://dx.doi.org/10.1890/04-1484

Moya N, Tomanova S, Oberdorff, T. 2007. Initial development of a multimetric index based on aquatic macroinvertebrates to assess streams condition in the Upper Isiboro-Sécure Basin, Bolivian Amazon. Hydrobiologia 589, 107-116. DOI: http://dx.doi.org/10.1007/s10750-0070725-3

Nkwoji JA, Yakub A, Ajani GE, Balogun KJ, Renner KO, Igbo JK, Ariyo AA, Bello BO. 2010. Seasonal Variations in the Water Chemistry and Benthic Macroinvertebrates of a South Western Lagoon, Lagos, Nigeria. J. Am. Sci., 6(3): 85- 92.

Okorafor KA, Andem AB, Okete JA, Ettah SE. 2012. The Composition, Distribution and Abundance of Macroinvertebrates in the Shores of the Great Kwa River, Cross River State, South-east, Nigeria. Eur. J. Zool. Res., 1(2): 31-36.

Ouéda A, Guenda W, Kabre AT, Zongo F, Kabré GB. 2007. Diversity, abundance and seasonal dynamic of zooplankton community in a southsaharan reservoir (Burkina Faso). J. Biol. Sci., 7(1): 1-9. DOI:

http://dx.doi.org/10.3923/jbs.2007.1.9

Ouedraogo I, Oueda A, Sirima D, Ouedraogo I, Guenda W, Kabré GB. 2015. Assessment of benthic molluscs diversity and distribution in urban reservoirs. Int. J. Biol. Chem. Sci., 9: 2066-2077. DOI: http://dx.doi.org/10.4314/ijbcs.v9i4.29

Ouedraogo R. 2010. Fish and fisheries prospective in arid inland waters of Burkina Faso, West Africa. PhD Thesis. University of Natural Resources and Life Sciences, Vienna, Austria, p. 222.

Sanwidi WJP. 2007. Groundwater potential to supply population demand within the Kompienga dam basin in Burkina Faso. $\mathrm{PhD}$ Thesis. Hohen Landwirtschaftlichen Fakultät Rheinischen FriedrichWilhelms-Universitätzu Bonn. http://hss.ulb.uni-bonn.de/diss_online elektronisch publiziert.

Sanogo S, Kabré T. 2014. Dynamique de structuration spatio-temporelle des populations de familles de macroinvertébrés dans un continuum lac de barrage-effluent-fleuve issu de périmètre irrigué. Bassin de la Volta (Burkina Faso). J. Appl. Biosci., 66306645 .

DOI: http://dx.doi.org/10.4314/jab.v78i0.9

Sanogo S, Kabré TJA, Cecchi P. 2014. Spatial-temporal dynamics of population structure for macro invertebrates families in a continuum dam - effluent - river in irrigated system. Volta Basin (Burkina Faso). Int. J. Agric. Policy Res., 2: $203-$ 214. DOI: http://dx.doi.org/10.4314/jab.v78i0.9

Sanogo S. 2014. Inventaire des macroinvertébrés de différents plans 
d'eau du bassin de la Volta en vue de l'identification des taxons bioindicateurs dans un continuum barrage hydroagricole-effluent-fleuve au Burkina Faso. Thèse de Doctorat. Université Polytechnique de Bobo Dioulasso, Burkina Faso, p. 200.

Sharma SR, Mathur MN, Saxena, S. Kaushik. 1993. Ecological studies of insect communities in the Saank, Asdun and Kunwari Rivers of Madhya Pradesh, India. Trop. Freshwater. Biol., 3: 287294.

Shelly SY, Mirza ZB, Bashir S. 2011. Comparative ecological study of aquatic macroinvertebrates of Mangla dam and Chashma barrage wetland areas. J. Anim. Plant Sci. 21: 340-350.

Tachet H, Richoux P, Usseglio-Polatera P. 2003. Invertébrés des Eaux Douces: Systématique, Biologie, Ecologie. Editions CNRS ; 119-148.

Tampo L, Ouéda A, Nuto Y, Kaboré I, Bawa LM, Djaneye-Boundjou G, Guenda W. 2015. Using physicochemicals variables and benthic macroinvertebrates for ecosystem health assessment of inland rivers of Togo. Int. J. Inn. Appl. Studies, 12(4): 961-976.

Uwadiae RE. 2013. Composition , Distribution and Diversity of Benthic Macroinvertebrates of Ona River, Southwest, Nigeria. Eur. J. Zool. Res., 1(2): 47-53.

Vinson MR, Hawkins CP, 1996. Effects of sampling area and subsampling procedure on comparisons of taxa richness among streams. J. North. Am. Benthol. Soc., 15(3): 392-399. DOI: http://dx.doi.org/10.2307/1467286

Voshell J, Reese J. 2002. A Guide to Freshwater Invertebrates of North America. Mc Donald \& Woodward Publishing Co.: Blacksburg, VA.

Wright IA, Chessman BC, Fairweather PG, Benson LJ. 1995. Measuring the impact of sewage effluent on the macroinvertebrate community of an upland stream. The effect of different levels of taxonomic resolution and quantification. Australian J. Ecol., 20(1): 142-149.

DOI: http://dx.doi.org/10.1111/j.14429993.1995.tb00528.x 